Review

\title{
A Review on Cognitive Radio for Next Generation Cellular Network and its Challenges
}

\author{
Nandhakumar Pandi and Arun Kumar \\ Electronics and Communication Engineering, JECRC University, Jaipur, India
}

\section{Article history}

Received: 11-02-2017

Revised: 06-03-2017

Accepted: 04-04-2017

Corresponding Author:

Nandhakumar Pandi

Electronics and

Communication Engineering,

JECRC University, Jaipur,

India

Email:

nandhakumar.1402062004@jec rcu.edu.in

\begin{abstract}
Cognitive Radio (CR) is considered an intelligent technology. It improves the utilization of the radio spectrum. The increasing number of new devices has resulted in growing congestion of the ISM bands. FCC issued an order only permitting unlicensed users (secondary users) to operate in white space without interfering with licensed users (primary users). Cognitive Radio Networks (CRNs) provides a solution to the problem of additional spectrum requirements by utilizing the spectrum more efficiently. $\mathrm{CR}$ is able to sense the spectrum and share the spectrum with unlicensed users. There are number of popular spectrum sensing techniques in CR such as cyclostationary feature detection, energy detection and matched filter detection, which capture the best available spectrum. They use a spectrum dynamically in wireless communication network. A CR with multicarrier technique (OFDM and FBMC) are the next generation of advanced cellular network, which would provide better spectrum utilization and therefore have greater capacity. In this study we have described and reviewed CR technology and its numerous features that may play a very essential role in the field of next generation wireless communication networks.
\end{abstract}

Keywords: Cognitive Radio Network, MC-CDMA, OFDM, FBMC, CR Challenges, CR Projects, Spectrum Sensing

\section{Introduction}

Wireless communication is the most important sector in communication media, how? In today's market, the whole world is depends on wireless communication system. According to the resent survey we find that around four billion mobile users are using wireless communication media. As the matter of fact, the cellular mobile have becomes a great business source and also users can fulfill their needs based on communication. Many equipment are there, which has to be controlled by wireless technology. Day-by-day, the accessibility of wireless devices are increasing because of the increased number of users. Due to the increased number of users, the spectrum utilization becomes more. So there is congestion in the bandwidth. Time Division Multiplexing (TDM) and Frequency Division Multiplexing (FDM) modules have used in third generation technology, but the problem with this system is, if the number of users are more, then congestion of bandwidth will arise. This problem has got sorted out by introducing Code Division Multiple Access (CDMA), which is the first multiple access technology, where in a single channel is capable of using multiple users. The next most advanced technology is multiple carrier technology is Multi-Carrier Modulation (MCM). In which, one carrier should be divided into multiple subcarriers. These sub-carriers will carry each information signal. For this operation each sub-carrier need separate modulators and demodulators, but this procedure is not possible practically. This process can be replaced by using Infinite Fast Fourier Transform (IFFT) operation at transmitter side and Fast Fourier Transform (FFT) operation at receiver side. The whole system will get simplified by introducing IFFT and FFT operations. This entire process is named as Orthogonal Frequency Division Multiplexing (OFDM) (Kumar and Gupta, 2015b). By implementing this on Digital Signal Processor (DSP), it is possible to operate the system at higher speed. In present scenario, the most advanced and promising is multi-carrier technology (OFDM). In this technology, the utilization of bandwidth is more as compared to conventional multi-carrier system. Due to the insertion of Cyclic Prefix (CP) in OFDM, it occupies 
some bandwidth. For example in LTE fixed 256 subcarrier system, $11.2 \%$ of bandwidth wasted due to the insertion of CP. To overcome from this problem one more technology has been introduced that is FBMC (Filter Bank Multi-Carrier). In this technology, there is no insertion of CP (Kumar and Gupta, 2015b). The next generation cellular network will be the Cognitive Radio Network (CRN), which will identify the unused bandwidth and allocate that bandwidth to the other users who needs. In cognitive network there will be two users, one is primary users and another is secondary users. These primary users are also named as licensed user and secondary users also named as un-licensed users. If primary users are idle, then the bandwidth of the primary user would be assigned to the secondary user (Haider and Ge, 2015; Padmavathi and Shanmugavel, 2015). In 2008, FCC gives out decision to utilize the free white space spectrum to the secondary user to use it without any interference to the primary users. Cognitive Radio (CR) has a capability of dealing with the spectrum issues than standard wireless environment face (Sun et al., 2015).

CR using a dynamic spectrum access method to accomplish more spectrum availability. CR decreases the inefficiencies caused by spectral congestion found in standard wireless environment, by giving adaptable application of the spectrum which are not used by primary users (Muthumeenakshi and Radha, 2014; Avila and Thenmozhi, 2015). One of the main processes of CRN is channel sensing. By using the dynamic spectrum access method to locate the free white space spectrum and assigned those free spectrum to the primary users (Akin and Gursoy, 2015). Pictorial representation of spectrum sensing is shown in Fig. 1.

In spectrum sharing, the good spectrum scheduling technique allows users to coexist on the same frequencies (Akyildiz et al., 2006). The best feature of $\mathrm{CR}$ is Re-configurability and ability. For the wireless environment, communication security is the main factor. Compare with the wired communication, wireless communication need more security to avoid the attacks from suspicious users. So, wireless system should be more privacy and integrity (Fragkiadakis et al., 2013).

The aim of CR technology is to boost up the throughput and minimize the impediment to primary users. One of the important areas in CR is spectrum in sensing. There are many spectrum sensing techniques in CR. They are:

- Interference based detection

- Co-operative detection

- Energy detection

- Cyclostationary detection

- Matched filter detection

Energy detection spectrum sensing is one of the detection techniques in CRN. It will give better performance only in higher SNR condition (Benitez and Casadevail, 2013). To predict the channel in CR is a challenging task. In CRN, secondary users are permitted to use the primary users channel without any interference. However, the channel quality may differ significantly and the good quality channel drastically decreases the spectrum efficiency for secondary user (Xing et al., 2013). In OFDM system, CP is added between the information to avoid the ISI (Inter Symbol Interference). Due to the insertion of CP in OFDM system, it occupies some bandwidth and it losses the efficiency of spectrum. To overcome this problem, CR should be integrated with the OFDM system.

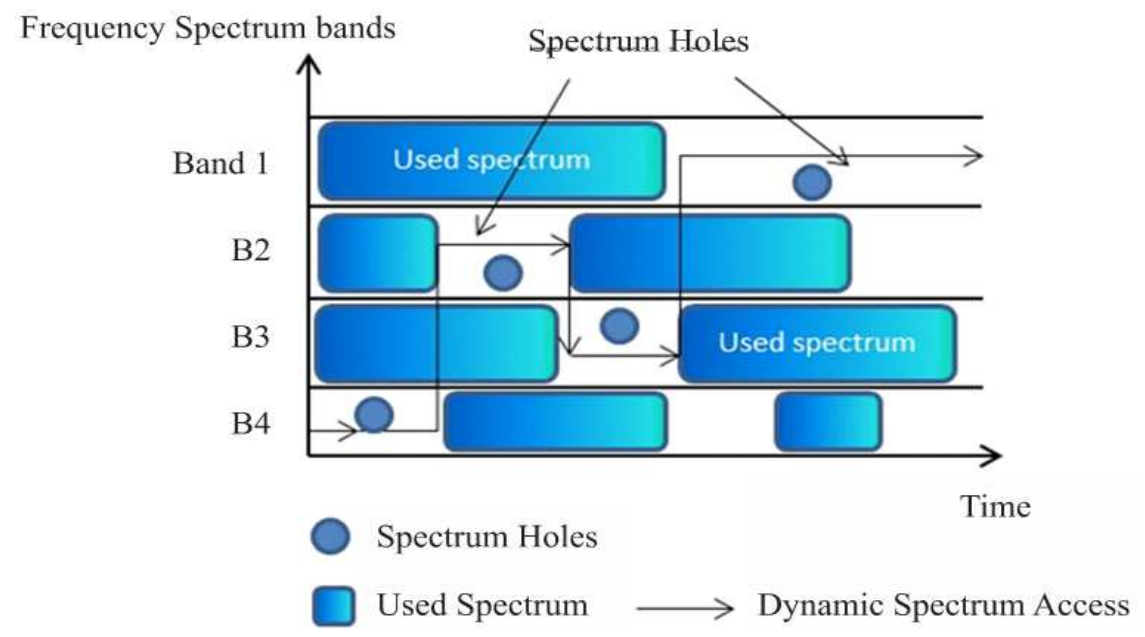

Fig. 1. Spectrum white space 
Cyclostationary feature detection is being considered with OFDM systems because it is a popular spectrum method and also it requires less Signal to Noise Ratio (SNR) for detection when compared to energy detection. Cyclostationary is a spectrum sensing method used in cognitive radio. It allows for detection of free portions of licensed user spectrum in its vicinity and it can also allocate that free spectrum to unlicensed users. Cognitive radio can detect the cyclostationary signal when the both licensed and unlicensed users are cyclostationary in nature with different frequencies. However, in lower order cyclostationary feature detection, it is very difficult to detect the presence of licensed users, when licensed and unlicensed users are in the same channel. Some techniques like higher order cyclostationary feature detection and Cyclo-energy detection can solve this spectrum sensing problem (Yang et al., 2012). Matched filtration is spectrum sensing method in cognitive radio. It is capable of detecting free portions of the primary user's spectrum and also allocates that free spectrum to secondary users. The advantage of using matched filtration is that it minimizes the spectral overcrowding problem. It is derived from cross correlating unknown signals with other known signals to detect the presence of well-known signals. It is also known to be an optimal linear filter. Matched filtration detection uses a dynamic threshold to improve the spectrum sensing efficiency and it also provides better performance in lower SNR (Kapoor et al., 2011; Salahdine et al., 2015).

\section{Cognitive Radio Background}

There has always been a continuous increase of user requirements in the cellular communication. This is what has led to the evolution of wireless technologies, exploring the future mobile systems. The four main aspects of cellular are radio access, bandwidth, data rates and switching schemes.

\section{Network Evolution}

The first ever Generation (1G) of cellular system was the Analog systems, which provided a bandwidth from 10 to $30 \mathrm{KHz}$, along with data rates ranging around 10 Kbps. Following it, the Second Generation (2G) was a GSM system. It had an increased bandwidth range of around $200 \mathrm{KHz}$, but with similar data rates to that of $1 \mathrm{G}$, i.e., nearly $9.6 \mathrm{Kbps}$. With the third Generation $(3 \mathrm{G})$ higher bandwidth and data rates were achieved i.e., $5 \mathrm{MHz}$ with $50 \mathrm{Mbps}$. Its successor; 4G cellular system provides much improved functionality as it can have a variable bandwidth of 20,40 or $70 \mathrm{MHz}$ with data rate ranging between $100 \mathrm{Mbps}$ to $1 \mathrm{Gbps}$ at the downlink. $4 \mathrm{G}$ is an advanced cellular system which comprises of LTE advanced and WIMAX advanced systems as well. The recent fifth Generation (5G) system also known as CR offers a new way of using the radio spectrum. It enables better frequency bands and wider spectral bandwidth per frequency channel. It enables a unique way of utilizing the free portion of a licensed spectrum by the unlicensed users. For example, if some area does not utilize a licensed broadcast spectrum, then that same spectrum can be used for mobile communication with the help of the adaptive nature of cognitive radio (Lee and Lee, 2010).

\section{Cognitive Radio with Multicarrier Technology}

In current applications, Multiple carrier technique is used, which is one the most advanced technologies. In this technique a single carrier is dived into multiple subcarriers. Thus the available bandwidths are the small limited bandwidths that were subdivided. Each of these subcarriers have a smaller bandwidth compared to the single carrier system. All the symbols to be transmitted are modulated with each carrier and transmitted through antennas to the respective channels, which is then demodulated by the demodulators at the receiving end. A transmitter block diagram of MCM is shown below in Fig. 2.

The process starts with passing the input symbols to the serial to parallel converter. There, $\mathrm{N}$ parallel symbols and ith data stream is modulated onto $i^{\text {th }}$ subcarrier. Thus, sum of all such subcarriers forms a composite signal which then would be transmitted on a channel. At the receiver end these signals are amplified first, following which it is passed on to the bank of demodulators or correlators. At this stage data is converted back to serial from parallel, successively forming a symbol stream. A receiver block diagram of an MCM is shown below in Fig. 3.

Thus it can be said that the MCM transmits $\mathrm{N}$ symbols in N/B time period using $\mathrm{N}$ subcarriers; where $B$ is the bandwidth given as Symbol rate $=N /(N / B)=B$. This ensures that the overall symbol rate in single carrier or multicarrier systems remains the same. In a similar manner, wideband system divides the bandwidth into narrow subcarriers in parallel, but implementation wise MCM has an upper hand because narrowband subcarriers experience frequency flat fading and bandwidth becomes less coherent. It is much easier to implement it in reliable detection schemes when the receiver and Inter Symbol Interference (ISI) are low. This is the core behind the operation of Multicarrier Modulation (MCM). It is very well understood that implementation of Bank of Modulators and Demodulators is challenging is a really challenging approach. Thus, instead of using N-Modulator and demodulators in MCM, we can simply generate it by IFFT operation, also known as OFDM. Pictorial representation of OFDM subcarriers is shown in Fig. 5 (Kumar and Gupta, 2015a). 


\section{Transmitter}

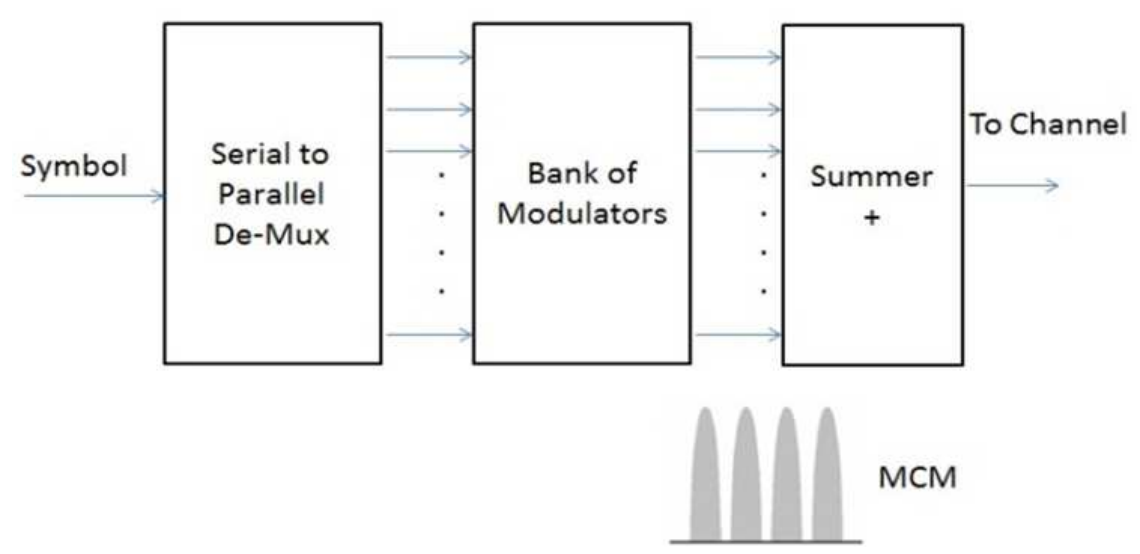

Fig. 2. Block diagram of MCM Transmitter

\section{Receiver}

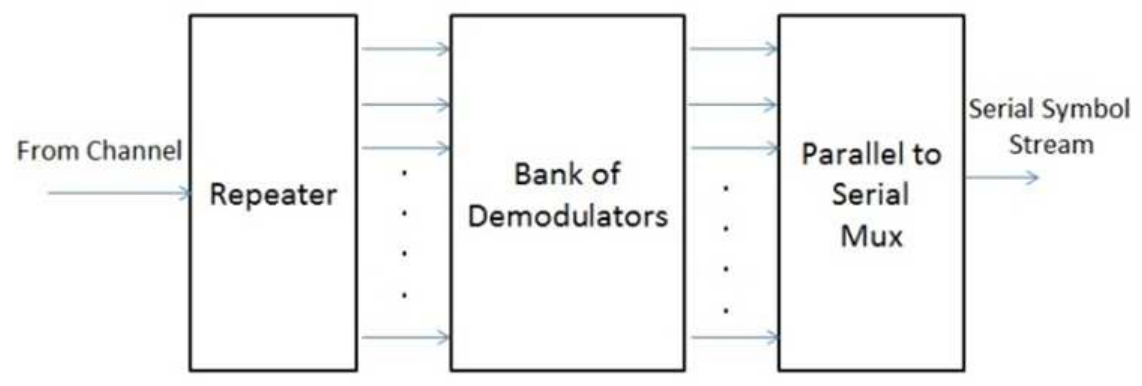

Fig. 3. Block diagram of MCM Receiver

\section{Transmitter}

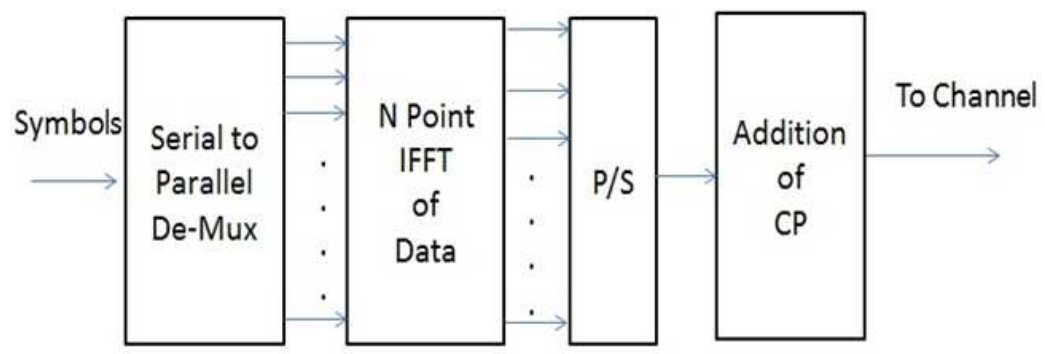

Receiver

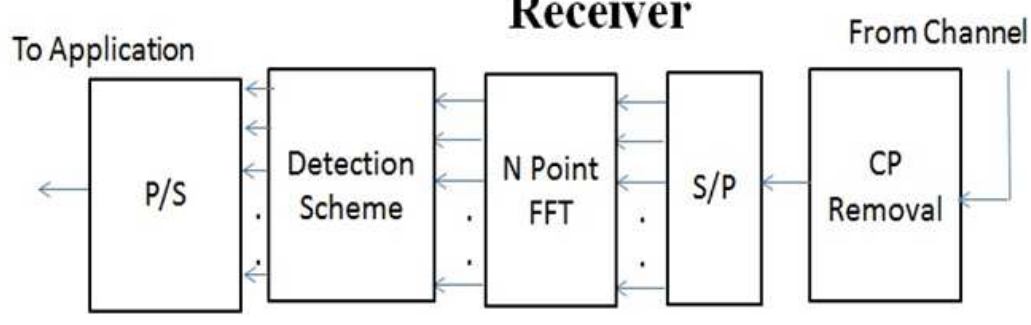

Fig. 4. Block diagram of OFDM Transmitter and Receiver. 


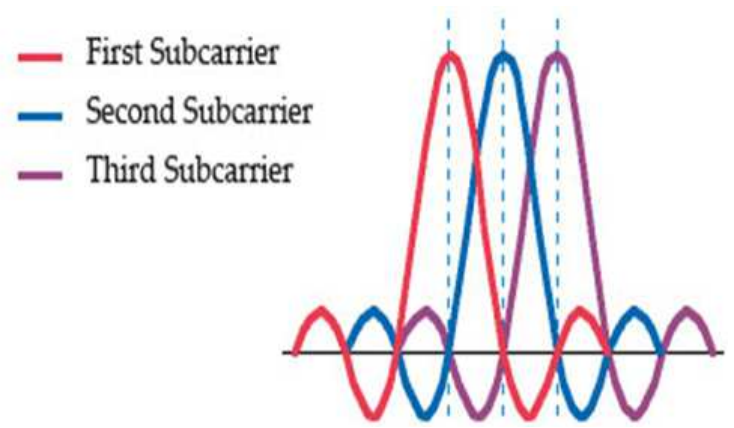

Fig. 5. OFDM Signal

\section{Orthogonal Frequency Division Multiplexing (OFDM)}

OFDM is a multicarrier modulation technique that divides a high data rate modulating stream to slowly modulated narrowband close-spaced subcarriers. It is less sensitive to frequency selective fading. OFDM has been adopted for many standards like Wi-Fi, Digital Video Broadcast (DVB) and DRM. The block diagram of an OFDM transmitter and receiver is shown below in Fig. 4.

The main advantages of the OFDM are its Immunity to selective fading, Spectrum efficiency and that it's free from ISI. However in cellular communication, the spectrum is not utilized properly which leads to Cognitive Radio (Kumar and Gupta, 2015b).

\section{Cognitive Radio with OFDM}

OFDM plays an important role in the current fourth generation network (LTE) and Wi-Max. Cognitive Radio solves the spectrum scarcity problem by using the TV white space spectrum. FCC issued an order to utilize the TV white space spectrum for unlicensed users. Implementing CR with OFDM technique provides efficient utilization of spectrum. OFDM is an exceptionally popular advanced technology, which is used in 4G (LTE) (Sun et al., 2013).

In cognitive Radio Network (CRN), there are three approaches, they are:

- Interweave CRN

- Underlay CRN

- Overlay CRN

\section{Interweave Cognitive Radio Network (CRN)}

In interweave CRN, the simultaneous access of spectrum to licensed and unlicensed user are not permitted, meaning: Only one user can access the spectrum at a time.

\section{Underlay Cognitive Network}

In this network, unlicensed users can share the licensed users channel continuously but the higher priority gives to licensed users only. This is known as spectrum sharing.

\section{Overlay Cognitive Network}

Secondary and primary users are permitted to transmit simultaneously with the help of coding. These are all the three main approaches in Cognitive Radio Network (Ekin et al., 2012).

\section{OFDM with Spectrum Sensing Model}

The input message symbols are stated as:

$$
b(n)=b(0) b(1) b(2) \ldots \ldots \ldots \ldots b(N-1) 0 \leq n \leq N-1
$$

These message symbols are converted from serial to parallel and IFFT is applied. This is stated as:

$$
B(n)=B(0) B(1) B(2) \ldots \ldots \ldots \ldots(N-1) 0 \leq n \leq N-1
$$

Therefore the complex OFDM modulated signal is specified by following mathematical form:

$$
P(t)=\sum_{K=0}^{N-1} B(t) k e^{j 2 \pi k \Delta t} 0 \leq t \leq T_{q}
$$

Where:

$P(t)=$ Composite OFDM modulated signal

$B(t)=$ IFFT of information signal

$\Delta t=$ Spacing between carriers

$T_{q} \quad=$ Symbol time

The signal is orthogonal if it satisfies the condition $T_{q} \cdot \Delta f=1$. To avoid the ISI, CP is added between the symbols. Therefore OFDM modulated signal with $\mathrm{CP}$ can be written as:

$P_{c p}(t)=\sum_{K=0}^{N-1} B(t) k e^{j 2 \pi k \Delta t t}+c p$

where, $P_{c p}(t)=$ Transmitted OFDM signal

The received signal is expressed as:

$S(t)=H(t) * P_{c p}(t)+N(t)$

Where:

$S(t)=$ Received signal across $t^{\text {th }}$ subcarrier

$H(t)=$ Channel co-efficient of $t^{\text {th }}$ subcarrier

$N(t)=$ Noise across $t^{\text {th }}$ subcarrier

The impulse response of the channel is given as:

$h(t)=\sum_{i=0}^{L-1} C_{i} \delta\left(t-\tau_{i}\right)$

Where: 


$$
\begin{aligned}
C_{i} & =\text { Attenuation factor } \\
\tau_{i} & =\text { Delay } \\
L & =\text { No. of Path }
\end{aligned}
$$

The received signal is given as:

$$
R(t)=S(t) * h(t)+n(t)
$$

Where:

$$
\begin{aligned}
& R(t)=\text { Received signal } \\
& h(t)=\text { Channel response } \\
& n(t)=\text { Noise of the channel }
\end{aligned}
$$

The spectrum detection technique is applied to the above received signal $R(t)$ and hypothesis is formulated that if the received signal $S_{c}$ is greater than the threshold, there will be detection of ideal spectrum otherwise no detection is considered:

$$
\begin{cases}x(t)=\{n(t)\} & H_{0} \\ x(t)=\left\{h * S_{c}(t)+n(t)\right\} & H_{1}\end{cases}
$$

Where:

$x(t)=$ Secondary user

$S_{c}(t)=$ Primary user's transmitted signal

$n(t)=$ AWGN

$h=$ Amplitude gain of the channel.

$H_{0} \quad=$ Absence of primary user signal

$H_{0} \quad=$ Presence of primary user signal

\section{Cognitive Radio with MC-CDMA}

The emerging high speed wireless communications are gradually increasing the demand for spectral resources. $\mathrm{CR}$ is one of the hopeful technologies to compensate this problem. MC- CDMA is being able to reduce ISI and Multiple User Interference (MUI) in frequency selective multipath fading channels. A cognitive radio with multicarrier CDMA is a new and well understood technology which provides a solution for spectrum scarcity problems (Dashti et al., 2012).

\section{$M C-C D M A$}

In a MC-CDMA, the transmitter spreads the original data over different subcarriers within its frequency domain, using a spreading code. Multiple SUs are assigned with orthogonal spreading codes to guarantee their separability at the receiver. CR networks use the subcarrier deactivation technique, which avoids the interference between MC-CDMA based SUs and PUs (Sundararajan and Govindaswamy, 2014). Multicarrier CDMA is a combination of CDMA and OFDM. It provides improved frequency diversity and higher data transfer rates. It offers a degree of flexibility in design (Sarath et al., 2007).

\section{CDMA with Spectrum Sensing Model}

The transmission of digital information is represented as $a_{k}(t)$ where $k=1 \ldots \ldots$ for $k$ users, is considered.

The data from the $k^{\text {th }}$ user are first modulated, before the spreading chip sequence $e_{k, i}(i=1 \ldots \ldots N)$ is applied over $N$ orthogonal subcarriers $f_{i}$.

The carrier frequency for the $i^{\text {th }}$ subcarrier, $f_{i}$ is expressed as:

$$
f_{i}=f_{1}+\frac{i-1}{T_{s}}
$$

where, $T_{s}=$ Symbol period.

The transmitted signal for the $k^{\text {th }}$ user is expressed by:

$$
G_{k}(t)=\sum_{i=1}^{N} \sqrt{2 P} a_{k}(t) e_{k, i} \cos \left(2 \pi f_{i} t+\varnothing_{k, i}\right)
$$

Where:

$$
\begin{aligned}
& P=\text { Signal power } \\
& \varnothing_{k, i}=\text { Random phase introduced by modulator }
\end{aligned}
$$

The received signal is written as:

$$
R(t)=H(t) G_{k}(t)+N(t)
$$

Where:

$R(t)=$ Received signal across $t^{\text {th }}$ subcarrier

$H(t)=$ Channel co-efficient of $t^{\text {th }}$ subcarrier

$G_{k}(t)=$ Transmitted MC-CDMA signal

$N(t)=$ Noise across $t^{\text {th }}$ subcarrier

The spectrum sensing detection technique is applied to the above received signal $R(t)$ and hypothesis is formulated that if the received signal is greater than the threshold, there will be detection of ideal spectrum otherwise no detection is considered:

$$
\begin{cases}x(t)=\{n(t)\} & H_{0} \\ x(t)=\left\{h^{*} R(t)+n(t)\right\} & H_{1}\end{cases}
$$

Where:

$x(t)=$ Secondary user

$R(t)=$ Primary user's transmitted signal

$n(t)=$ AWGN

$h=$ Amplitude gain of the channel

$H_{0}=$ Absence of primary user signal

$H_{0}=$ Presence of primary user signal

\section{Advantages of MC-CDMA with CR}

It provides a enhanced capacity for voice and data communication and it allows the subcarrier to occupy the same frequency at the same time on a given band. 
CDMA is a $3 \mathrm{G}$ network. CDMA is a superior choice for cellular environments and a cognitive radio with CDMA technique will be the best choice for future wireless environments (Roy and Kundu, 2010).

\section{Cognitive Radio with Filter Bank Multicarrier (FBMC)}

Multicarrier modulation is a promising technique for wireless communication; it allows high data transmission rate over parallel sub-channels. OFDM is an attractive and advanced technique, which is able to avoid ISI and ICI by adding a CP (Chen et al., 2010).

\section{Problems in OFDM}

The spectral outflow in conventional OFDM due to the large side lobes decrease the performance of OFDM based on cognitive radio network systems. The $\mathrm{CP}$ in OFDM holds redundant data which decreases the spectral efficiency (Sundararajan and Govindaswamy, 2014) and it also causes high mutual interference between Primary User's due to its large frequency domain side- lobes. The total spectrum efficiency is despoiled due to the insertion of $\mathrm{CP}$ to each of the OFDM symbols (Absi and Kaiser, 2014). To compensate for this problem, another promising multicarrier technique is the Filter Bank Multicarrier (FBMC).

\section{FBMC VS OFDM}

FBMC doesn't require any $\mathrm{CP}$ and it provides more robustness to the frequency and time offset than OFDM. It also offers high spectrum resolution and high data transition rates by providing independent sub-channels. FBMC is resultant from a prototype filter that gives the level of attenuation for each of the other sub channels. Cognitive Radio with OFDM undergoes high interference to the primary users due to its large side lobes' frequency response to filter. But in FBMC with the Offset Quadrature Amplitude Modulation (OQAM) it can achieve reduced Inter Carrier Interference (ICI) and ISI without using the CP. So the difficult of the spectral outflow can be solved by reducing the effect of the side lobes of each subcarrier, which leads to higher spectral efficiency (Shaat and Bader, 2012). The FBMC based CR system has more capability than the OFDM based system. FBMC will be a future Cognitive Radio system, which provides better spectrum utilization.

\section{Cognitive Radio with Ultra Wide Band (UWB)}

Nowadays, the number of devices adapting to wireless communication is increasing. In the future, it will be difficult to allocate the radio spectrum for those devices. To compensate for this spectrum problem,
Cognitive Radio systems can be used, it is needed to detect the unused spectrum over a wide-ranging frequency and modify its working parameters consequently to attain extraordinary spectral efficiency. There are two frequency bands where the Cognitive Radio might operate in the future, they are:

- $\quad$ UHF TV bands (400-800 MHz)

- Long and short range application band (3-10 GHz) (Hayar, 2011)

\section{Present Related Work in CUWB}

A Super-Regenerative Band- Pass (SR-BP) filter in RF spectrum detecting system was proposed by (Anis et al., 2010) to provide low power, low price and short range data transmission rate CUWB. SB-BP filters are used in both narrowband and wideband sensing schemes to detect the spectrum. UWB systems transfer the data by using the very short duration pulses and possess a large bandwidth. To merge the CR with UWB technologies, UWB system will have to be able to Co-exist with the narrowband licensed system. The aim of the CR is to sense the licensed user and adjust their communication parameters to escape from interference with the primary side. UWB system achieves the spectrum detecting technique to choose the best suitable transmission and receiver filter, which will reduce the fault rate in performance (Khamy et al., 2013). Combining the CR and UWB detects the efficient RF spectrum without any interference to primary users (Sarabchi and Nerguizian, 2010).

\section{Advantages of UWB with $C R$}

The main advantage of developments in Ultra Wide Band (UWB) cognitive radio is that capacity can be maximized, while reducing interference and that the spectrum can be shared more efficiently. This is the foundation of designing a Software Defined Radio (SDR) (Sablatash, 2007).

\section{Projects on Cognitive Radio}

$\mathrm{CR}$ is a novel method of thinking on wireless communication. It is a main applicant technology for the $4 \mathrm{G}$ wireless system. The main motive of this technology is to improve utilization of the spectrum. Several agencies are already studied about the current usages of the radio spectrum and numerous researches have been initiated. Five European schemes of cognitive radio topic were endorsed with full budget of more than $€ 50$ million (Benedetto et al., 2008). They are:

- ARAGORN

- EUWB

- PHYDYAS

- E3 


\section{Adaptive Reconfigurable Access and Generic Interfaces for Optimization in Radio Networks (ARAGORN)}

The main goal of this technology is to increase the system performance and efficiency of the current day and future systems, as well as subsequent cognitive radio and network paradigms.

\section{Objectives of ARAGORN:}

- To develop machine learning based tool in order to manage the complexity of the optimization of the communication stack

- To design the architecture for the Cognitive Radio Manager (CRM) and enabling CRM to arbitrate between different, optimization goals, often conflicting in multi-dimensional reasoning process

- To provide the interfaces required to enable the CRM

- To prototype the scheme on reconfigurable hardware with new software management

This project will combine the CRM and related software modules. This experiment is performed in the $2.4 \mathrm{GHz}$ ISM band (Mahonen et al., 2009).

\section{European Research on Ultra Wide Band (EUWB)}

The main aim of this project is to consolidate technical advance in scientific areas related to Ultra Wide Band (UWB), such as cognitive signaling, intelligent multiband antenna and multimode concepts. Based on the strong demand from the European industry sector, the four prominent examples are implemented, they are:

- Automotive environment (driven by Bosch)

- Public transport environment (driven by EADS)

- Intelligent home environment (driven by Philips)

- Next generation of heterogeneous public access network environment (driven by Telefonics)

Project started on 1-04-2008 and its ends on 31-072011. The total cost of the project is $20,990,941 €$. It provides a new and next generation network with ultra high speed short range wireless access (EUWB, 2008).

\section{Physical Layer for Dynamic Spectrum Access (PHYDAS)}

Physical layer is best suited to the new concept of $\mathrm{CR}$ and Dynamic Spectrum access Management (DSM). In future mobile radio network, high data rates and flexible spectrum allocation can be done by the multicarrier technique. Here FBMC offers high spectrum resolution as it provides an independent subchannels (Maurice Bellanger, 2010).

\section{Objective of PHYDAS}

To develop and demonstrate algorithm for single and multi-antenna terminals, adaptivity and stability and multiple access. The impacts on cognitive radio are also investigated.

\section{End to End Efficiency (E3)}

End to End efficiency project is a huge scale integrating development. The aim of this project is to integrate cognitive wireless system in the Beyond $3 \mathrm{G}$ (B3G) world.

\section{Objective of E3}

To plan, extend prototype and platform solution to assurance interoperability, plasticity and scalability between vacant ligancy and future wireless system.

E3 plan of next invention of different standards (IEEE 802.16/11, P1900, ETSI, 3GPP) allowing a flawless employ of these standards to fulfill the situations of the existing $4 \mathrm{G}$ system at lesser price and better spectrum efficiency (E3, 2008).

\section{Spectrum Pricing for Cognitive Radio (CR)}

This project was proposed by Oloyede $\mathrm{A}$ and Grace D on 2011. Many technologies and devices such as Radio, TV broadband, CCTV, sensor, alarm system, remote control and other devices requires the radio spectrum. Although, it increases the number of devices using the spectrum, it fails to improve the utilization due to the static portion of the band. The aim of this project is to achieve the DSM using a Cognitive Radio system.

European commission funded to improve consistency collision of study schemes on CR and spectrum sensing because there is a need to fulfill demands in capability and exposure. CR is a capable method for dynamic spectrum sharing. In Europe, UHF TV spectrum is not used by the broadband services and Cognitive radio technique utilizes the TV spectrum for mobile network (Marques et al., 2013).

\section{Challenges in Cognitive Radio}

An increasing number of services and application devices operating on radio communication are rapidly consuming the spectral resources. Providing a fixed portion of spectrum, certain services lead to poor spectrum utilization. Some licensed portions of the spectrum are unused at times in some specific geographical areas. Those spectrums are referred to as spectrum holes, which cannot be used by any other user in current fixed spectrum allocation systems. It's possible to utilize these spectrum holes by using the concept of Cognitive Radio. It improves the resource usage efficiency in communication systems. Cognitive 
Radio uses a dynamic spectrum access (Tarchi et al., 2014). There are plenty of challenges in designing the Cognitive Radio. The popularity of smart phones is increasing rapidly so there is a lack of available spectrum for these devices to access. Thus, there are lots of problems arising in spectrum availability. Which leads to the use the spectrum otherwise left to TV bands up to 60 GHz. This is called as TVWS. In order to address the problem of spectrum usage efficiency, CR concept was proposed (Nguyen et al., 2013).

\section{Research Challenges}

There is plenty of research going on regarding cognitive radio technology:

- The first research issue is how to make the decisions. Decision making is the biggest challenge in Cognitive Radio Network (CRN)

- Choice of the decision algorithm. Many algorithms were available for decision making, such as genetic algorithm, neural network, etc. which will fulfill the cognitive radio system requirements

- The design of MAC sub- layer and network layer for cognitive networks is, itself a challenging job

- Security is one of the issues in Cognitive Radio Network

\section{Implementation Challenges}

To implement a CR is a challenging task. CR should familiarize transmission and reception to avoid interference with primary users. There are numerous techniques can be used to avoid the interferences and combine such frequency tuning. (Nguyen et al., 2011), they are:

- Adaptive frequency hopping

- Dynamic frequency selection

- $\quad$ RF band switching

The another challenges of $\mathrm{CRN}$ are to monitor the environment and then achieve the resources logically based on practices and also there are three important challenges in implementing a Cognitive Radio, they are:

- $\quad \mathrm{RF}$ front end-transceiver challenges

- $\mathrm{ADC}$ and DAC challenges

- Baseband challenges

Cognitive radio has to face very challenging issues to perform sustainable communication. The technical challenges are designing the RF- front end, ADC, DAC and to make it able to support flexible wideband multiband communication (Hayar et al., 2007).

\section{Security Challenges in CRN)}

The continuous development of CR during the last decade has significantly fascinated the research and development in the industry and also in academia. In any wireless communication security is an important aspect. In a traditional wireless network, security attacks are the most problematic one. There are two main classes of security issues in CRN (Attar et al., 2012), they are:

- Traditional security threats

- $\mathrm{CRN}$ - Specific threats

The classifications of security attacks in CRN are Infrastructure based and Infrastructure less Cognitive Radio Network specific attacks.

\section{Infrastructure Based CRN Attacks}

An infrastructure based cognitive radio network attacks are time consuming and expensive. Here the CRNs will practically be adjusted towards frequency bands with second priority spectrum stability. There are many attackers in the infrastructure based $\mathrm{CRN}$, they are:

- IE (Incumbent Emulation)

- SSDF (Spectrum Sensing Data Falsification)

- Control channel jamming

\section{Infrastructure-Less CRN Specific Attacks}

In infrastructure-less CRN specific attacks, there are three main attackers, they are:

- Exogenous Attacker

- Intruding Attacker

- Greedy CRS

\section{Exogenous Attacker}

It is not a portion of the Cognitive Radio Network and thus not part of the CRN's spectrum detecting resolution. This attacker can disturb the process of the ad-hoc CRN over jamming attacks.

\section{Intruding Attackers}

Ad-hoc CRNs are vulnerable to intruding challenger nodes attack which can enter into the system pushing as authorized nodes. This cruel node can influence the overall spectrum sensing decision of CRN. This class of CRN security issue is known as SSDF.

\section{$S S D F$}

SSDF stands for Spectrum Sensing Data Falsification. This is one type of attack in CRNs which causes perpetual reporting of a busy channel. To identifying this type of attack is very difficult. 


\section{Jamming}

It is a most widely used to attack wireless transmissions. It reduces the received SNR below the needed threshold by transmitting noise over the receiving channel (Attar et al., 2012).

\section{Challenges in CR for Disaster Response Networks}

Disasters are unexpected or unplanned events that damage the life. If any disaster strikes, it will damage the network and also increased traffic demand. So the responder cannot receive the information they need and also they cannot report their location. Quick repair of the network is not possible and it is critical after the disaster. There is a need to install a new flexible infrastructure that can provide an emergency service in the disaster place and start to help the people. Many services like $2 \mathrm{G}, 3 \mathrm{G}$ and $4 \mathrm{G}$ mobile systems need support. To access the available radio spectrum to communicate with them, is a biggest challenge. $\mathrm{CR}$ is the only knowledge that can intelligently sense the environment and make decisions and reconfigure itself in response to these observations and learn from its experience (Ghafoor et al., 2014). The multiple CRs form a Cognitive Radio Network and it can adapt to its environment. It supports for heterogeneity, self-organization, re-configurability and interoperability with existing networks. It can also provide a solution for disaster response.

\section{Types of Spectrum Sensing in CR}

The competent method to identify the spectrum holes is to identify the main users that are receiving information within communication choice. The spectrum sensing methods are classified as:

- Transmitter detection (Non cooperative detection)

- Energy detection

- Cyclostationary detection

- Matched filter detection

- Cooperative detection

- Interference based detection

The best standard spectrum detection techniques are:

- Cyclostationary feature detection

- Energy detection

- Matched filter detection

\section{Spectrum Sensing Model}

To detect the spectrum holes in cognitive radio by sensing the spectrum, each secondary user chooses between two subsequent hypotheses that symbolize the presence $\left(H_{1}\right)$ and absence $\left(H_{0}\right)$ of licensed user signal in the licensed frequency band. Mathematically it can be represented as:

$$
\left\{\begin{array}{l}
H 0: \quad x_{i}(t)=n_{i}(t) \\
H 1: x_{i}(t)=h_{i} s(t)+n_{i}(t) i=1, \ldots N_{u}
\end{array}\right.
$$

Where:

$x_{i}(t)=$ Represents the $i^{\text {th }}$ Second priority user's received signal

$N_{u}=$ Represents the number of Second priority users

$S(t)=$ Represents the licensed users transmitted signal

$n_{i}(t)=$ Represents the AWGN

$h_{i}=$ Represents the gain of the channel between PU and $i^{\text {th }} \mathrm{SUs}$

\section{Cyclostationary Detection}

Cyclostationary detection is an alternative detection method. Cyclostationary method is an arbitrary procedure for which the data, like mean and autocorrelation, vary occasionally with time. Modulated signals are combined with carriers, pulse trains, hopping series or repeated prefixes and repeating spreading. These modulated signals are distinguished as cyclostationary. Cyclostationary detection method is used at very smaller SNR condition. It is robust to noise, carry out better than many sensing schemes like energy power but it has greatly extended computational complication (Sansoy and Buttar, 2015). Cyclostationary spectrum detection method performs as a satisfactory way when compared to other detection schemes, because it has the capability of rejection of noise. At the same time, it has some disadvantages too. These include spectral leakage of high amplitude signals, non- linearity and also high costs (Mohapatra et al., 2013).

\section{Energy Detection}

Energy detection is a standard scheme for detecting the spectrum and it detects the occurrence of spectrum holes and improves the detection possibility. Block diagram of energy detection process is shown in Fig. 6.

Here the received signal is transformed from time to frequency by FFT operation then PSD is determined and the signal is sent to a band pass filter to choose the correct channel where it is integrated over time.

The output of the energy detector is compared with the threshold $(\lambda)$ and it decides the hypothesis

$$
E \geq \lambda, E \leq \lambda
$$




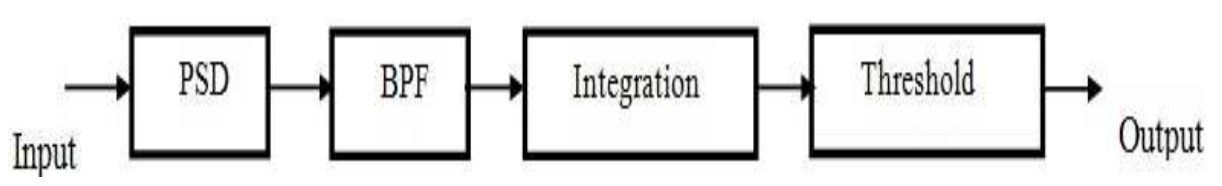

Fig. 6. Energy detection process

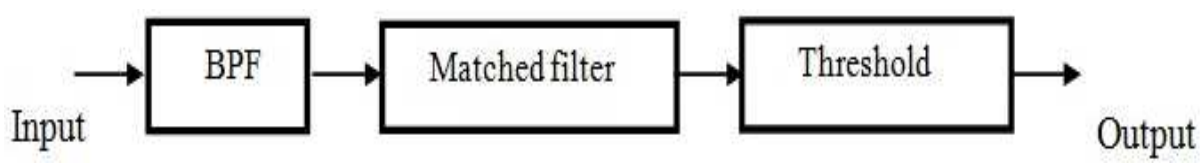

Fig. 7. Block diagram of matched filter detection

The threshold can be constant or able to change depending on the conditions of the channel. However this method is inaccurate (Sansoy and Buttar, 2015). Energy detection is the best scheme for detecting independently distributed signals of high SNR condition, but it's not suitable for detecting correlated signals. There are three parameters for defining the presence and absence of the signal:

- $\quad P_{m}$ (Probability of missed detection)

- $\quad P_{f}$ (Probability of false alarm)

- $P_{d}$ (Probability of detection)

A high $P_{f}$ results in low spectrum utilization (Pandya et al., 2015).

\section{Matched Filtration Detection}

Matched filtration is performed by a linear filter which gives maximum SNR with a given transmitted signal. It needs the exact synchronization and the existing information of PU's features such as BW, modulating type, operating frequency and pulse shaping. The decision can be done by whether the signal is present or not can be known if we pass the signal through a filter, which will stress the useful signal and quash the noise at the same time this will give a sharp difference between the signal and the noise.

If a signal is present, the output will come out to have a large peak at this instant, if not, no peak will appear. The block diagram of matched filter detection is shown in Fig. 7.

Input is band passed, correlated with the time shifted threshold value $(\lambda)$, if the output is greater than $\lambda$, then PU is present $\left(H_{1}\right)$, otherwise $H_{0}$.

\section{Future Scope}

Day by day the importance of cellular communication is increasing and people want to connect to a better quality network at all times, everywhere. OFDM has a lot of advantages over conventional multicarrier techniques with regards to spectrum efficiency and reduced Inter Symbol Interference (ISI). In OFDM, Cyclic Prefix (CP) is used to reduce the interferences between the symbols, but high side-lobes in subcarriers cause Inter Carrier Interference (ICI). This is one of the major problems for OFDM. In OFDM and FBMC, reduction of ICI is a complex task. Universal Filter Multicarrier (UFMC) is one of the multicarrier transmission schemes, it uses a sub-band filter to each subset of subcarriers to reduce the effect of the sidelobes and it can also reduce the ICI in a better manner. Cognitive Radio is being considered as a next generation wireless system, it will be integrated with UFMC which will be a key candidate for the fifth Generation $(5 \mathrm{G})$ wireless system. It will provide better spectrum utilization and it provides better performance when compared with OFDM and FBMC. Another promising future technology for next generation of cellular communication is Massive MIMO integrated with Cognitive radio. In the present scenario, switching from one network to another network (Vertical Handover) is not an easy task; there will be a delay experienced in switching, most of the times call or data drops occur. Nowadays, all devices are compatible with $2 \mathrm{G}, 3 \mathrm{G}$ and $4 \mathrm{G}$ networks so switching from one network to another network automatically occurs whenever any network is available in order to maintain communication. Bandwidth is the important factor considered in vertical handover. In order to provide better handover with QoS, there will be a need to manage bandwidth necessity and power consumption during handover. Cognitive Radio will be the future technology which will reduce the bandwidth constraint and delay for vertical handover and it will also provide better QoS.

\section{Conclusion}

From the study we come to a conclusion that, demand for spectrum in wireless environment has fully grown substantially due to the widespread use of smart phones. We have proposed a cognitive radio which senses and splits the spectrum between the licensed and unlicensed users while increasing the performance of 
the network. Cyclostationary detection is the best spectrum sensing technique, it can sense a spectrum even in low SNR conditions and other spectrum detecting techniques like energy detection and matched filter detection are also available for detection of spectrum. A cognitive radio with multicarrier techniques (OFDM and FBMC) are also being proposed, which provides better spectrum utilization and improvement in transmission performance. The multi-carrier technology with cognitive radio will be implemented with better BER aim at maximum utilization of bandwidth. In future, it will be a great idea to rent our bandwidth when it is not in use with anyone who is in-need of it: This will efficiently utilize the spectrum and shortage of bandwidth will be solving in greater extend.

\section{Acknowledgement}

Author's like to thank to JECRC University for support this research. The first author also thanks to Dr. B. Ranjith (Post-doctoral Researcher) for his support and valuable suggestions.

\section{Author's Contributions}

Nandhakumar Pandi: Has conducted the research and written the paper.

Arun Kumar: Has designed the research plan.

\section{Ethics}

The work done in this paper is original and has not been submitted anywhere else.

\section{References}

Avila, J. and K. Thenmozhi, 2015. Upgraded spectrum sensing method in cognitive radio network. Indian J. Sci. Technol., 8: 1-4. DOI: $10.17485 / \mathrm{ijst} / 2015 / \mathrm{v} 8 \mathrm{i16} / 57499$

Akin, S. and C. Gursoy, 2015. Performance analysis of cognitive radio systems with imperfect channel sensing and estimation. IEEE Trans. Commun., 63: 1554-1566.

DOI: 10.1109/TCOMM.2015.2412539

Akyildiz, I.F., W.Y. Lee, M.C. Vuran and S. Mohanty, 2006. Next generation/dynamic spectrum access cognitive radio wireless network-A survey. Comput. Netw., 50: 2127-2159.

DOI: $10.1016 /$ j.comnet.2006.05.001

Attar, A., H. Tang, A.V. Vasilakos, F.R. Yu and V. Leung, 2012. A survey of security challenges in cognitive radio networks: Solutions and future research directions. Proc. IEEE, 100: 3172-3186. DOI: 10.1109/JPROC.2012.2208211
Anis, M., M. Ortmanns and N. When, 2010. RF spectrum sensing technique for cognitive UWB radio network. Sept. 14-16, Proceedings of the ESSCIRC, IEEE Xplore Press, pp: 506-509. DOI: 10.1109/ESSCIRC.2010.5619754

Absi, M. and T. Kaiser, 2014. Optimal resource allocation based on interference alignment for OFDM and FBMC MIMO cognitive radio systems. Proceedings of the European Conference on Networks and Communications, Jun. 23-26, IEEE Xplore Press, pp: 1-5. DOI: $10.1109 /$ EuCNC.2014.6882676

Benitez, M. and F. Casadevail, 2013. Signal uncertainty in spectrum sensing for cognitive radio. IEEE Trans. Commun., 61: 1231-1241. DOI: 10.1109/TCOMM.2013.021413.110807

Benedetto, D.M., Y. Hua, T. Kaiser and X. Wang, 2008. Cognitive radio technology. IEEE Signal Process. Magaz., 25: 10-198. DOI: 10.1109/MSP.2008.930499

Chen, D., D. Qu and T. Jiang, 2010. Novel prototype filter design for FBMC based cognitive radio systems through direct optimization of filter coefficients. Proceedings of the International Conference on Wireless Communications and Signal Processing, IEEE Xplore Press, pp: 1-6. DOI: $10.1109 /$ WCSP.2010.5633530

Dashti, M., P. Azmi and K. Navaie, 2012. Resource allocation for underlay CDMA cognitive radio networks. Proceedings of the IEEE Wireless Communications and Networking Conference, Apr. 1-4, IEEE Xplore Press, pp: 2792-2976. DOI: $10.1109 /$ WCNC.2012.6214276

Ekin, S., M.M. Abdallah, K.A. Qaraqe and E. Serpedin, 2012. Random subcarrier allocation in OFDM-based cognitive radio networks. IEEE Trans. Signal Process., 60: 4758-4774. DOI: 10.1109/TSP.2012.2203126

EUWB, 2008. European research on ultra wideband. R $\&$ D.

E3, 2008. End to end efficiency.

Fragkiadakis, A.G., E.Z. Tragos and I.G. Askoxylakis, 2013. A survey on security threats and detection techniques in cognitive radio networks. IEEE Commun. Surveys Tutorials, 15: 428-445. DOI: 10.1109/SURV.2011.122211.00162

Ghafoor, S., P. Sutton, C. Sreenan and K. Brown, 2014. Cognitive radio for disaster response networks: Survey, potential and challenges. IEEE Wireless Commun., 21: 70-80. DOI: 10.1109/MWC.2014.6940435

Haider, F. and X. Ge, 2015. Spectral and energy efficiency analysis for cognitive radio networks. IEEE Trans. Wireless Commun., 14: 2969-2980. DOI: 10.1109/TWC.2015.2398864 
Hayar, M., R. Pacalet and R. Knopp, 2007. Cognitive radio research and implementation challenges. Proceedignss of the Conference Record of the 41th Asilomar Conference on Signals, Systems and Computers, IEEE Xplore Press, pp: 782-786. DOI: 10.1109/ACSSC.2007.4487323

Hayar, A., 2011. Some issues on cognitive radio and UWB technology convergence for enabling green networks. Proceedings of the 7th International Workshop on Systems, Signal Processing and their Applications, May 9-11, IEEE Xplore Press, pp: 365-370. DOI: 10.1109/WOSSPA.2011.5931513

Kumar, A. and M. Gupta, 2015a. Key technologies and problems in deployment of $5 \mathrm{G}$ mobile communication system. Commun. Applied Electron., 1: 2394-4714.

Kumar, A. and M. Gupta, 2015b. A review on OFDM and PAPR reduction techniques. Am. J. Eng. Applied Sci., 8: 202-209. DOI: 10.3844 /ajeassp.2015.202.209

Kapoor, S., S. Rao and G. Singh, 2011. Opportunistic spectrum sensing by employing matched filter in cognitive radio network. Proceedings of the International Conference on Communication Systems and Network Technologies, Jun. 3-5, IEEE Xplore Press, pp: 580-583.

DOI: $10.1109 /$ CSNT.2011.124

Khamy, R., S. Shaaban, I. Ghaleb and H.N. Kheirallah, 2013. An Interference-Aware Cognitive Radio UWB (IA-CR-UWB) system using Eigen pulse shaping. Proceedings of the International Conference on Technological Advances in Electrical, Electronics and Computer Engineering, May 9-11, IEEE Xplore Press, pp: 16-21.

DOI: 10.1109/TAEECE.2013.6557188

Lee, C. and W. Lee, 2010. Exploiting spectrum usage patterns for efficient spectrum management in cognitive radio networks. Proceedings of the 24th IEEE International Conference on Advanced Information Networking and Applications, IEEE Xplore Press, pp: 320-327.

DOI: $10.1109 /$ AINA.2010.53

Muthumeenakshi, K. and S. Radha, 2014. Optimal techniques for sensing error minimization with improved energy detection in cognitive radios. Int. J. Smart Sens. Intell. Syst., 7: 2014-2034.

Mohapatra, S., A.G. Mohapatra and S.K. Lenka, 2013. Performance evaluation of cyclostationary based spectrum sensing in cognitive radio network. proceedings of the International Multi-Conference on Automation, Computing, Communication, Control and Compressed Sensing, Mar. 22-23, IEEE Xplore Press, pp: 90-97.

DOI: $10.1109 /$ iMac4s.2013.6526389
Maurice Bellanger, 2010. Physical layer for dynamic spectrum access and cognitive radio PHYDAS.

Marques, P., J. Rodriguez, S. Delaere, P. Delahaye and B. Lecroart et al., 2013. Shared use of radio spectrum in the EU: From research projects to standards. Proceedings of the IEEE International Conference on Communications Workshops, Jun. 913, IEEE Xplore Press, pp: 1249-1254. DOI: 10.1109/ICCW.2013.6649428

Nguyen, V., F. Villain and Y. Le Guillou, 2011. Cognitive radio systems: Overview and challenges. Proceedings of the 3rd International Conference on Awareness Science and Technology, Sept. 27-30, IEEE Xplore Press, pp: 497-502. DOI: 10.1109/ICAwST.2011.6163179

Padmavathi, G. and S. Shanmugavel, 2015. Performance analysis of cooperative spectrum sensing technique for low SNR regime over fading channels for cognitive radio networks. Ind. J. Sci. Technol., 8. DOI: $10.17485 / \mathrm{ijst} / 2015 / \mathrm{v} 8 \mathrm{i} 16 / 64746$

Pandya, P., A. Durvesh and N. Parekh, 2015. Energy detection based spectrum sensing for cognitive radio network. proceedings of the 5th International Conference on Communication Systems and Network Technologies, Apr. 4-6, IEEE Xplore Press, pp: 201-206. DOI: 10.1109/CSNT.2015.264

Mahonen, P., M. Petrovaa and J. Nasreddine, 2009. Adaptive reconfigurable access and generic interfaces for optimization in radio networks.

Roy, S. and S. Kundu, 2010. Performance of an adaptive power based CDMA cognitive radio networks. Proceedings of the IEEE Symposium on Industrial Electronics and Applications, Oct. 3-5, IEEE Xplore Press, pp: 28-33. DOI: $10.1109 /$ ISIEA.2010.5679502

Sun, L., W. Zheng, N. Rawat, V. Sawant and D. Koutsonikolas, 2015. Performance comparison of routing protocols for cognitive radio networks. IEEE Trans. Mobile Comput., 14: 1272-1286. DOI: 10.1109/TMC.2014.2346782

Sun, S., J. Yanhong and Y. Yamao, 2013. Overlay cognitive radio OFDM system for $4 \mathrm{G}$ cellular networks. IEEE Wireless Commun., 20: 68-73. DOI: 10.1109/MWC.2013.6507396

Sundararajan, M. and U. Govindaswamy, 2014. Multicarrier spread spectrum modulation schemes and efficient FFT algorithms for cognitive radio systems. Electronics, 3: 419-443. DOI: 10.3390/electronics3030419

Sarath, D., K.E. Nolan, P.D. Sutton and L.E. Doyle, 2007. Exploring the reconfigurability options of multi-carrier CDMA in cognitive radio systems. Proceedings of the IEEE 18th International Symposium on Personal, Indoor and Mobile Radio Communications, Sept. 3-7, IEEE Xplore Press, pp: 1-5. DOI: 10.1109/PIMRC.2007.4394698 
Sansoy, M. and A. Buttar, 2015. Spectrum sensing algorithms in cognitive radio: A survey. Proceedings of the IEEE International Conference on Electrical, Computer and Communication Technologies, Mar. 5-7, IEEE Xplore Press, pp: 1-5.

DOI: 10.1109/ICECCT.2015.7226181

Shaat, M. and F. Bader, 2012. Comparison of OFDM and FBMC performance in multi-relay cognitive radio network. proceedings of the International Symposium on Wireless Communication Systems, Aug. 28-31, IEEE Xplore Press, pp: 756-760. DOI: 10.1109/ISWCS.2012.6328469

Sarabchi, F. and C. Nerguizian, 2010. Interference cancellation technique for MIMO MB-OFDM UWB cognitive radio. Proceedings of the 6th International Conference on Wireless and Mobile Communications, Sept. 20-25, IEEE Xplore Press, pp: 472-277. DOI: 10.1109/ICWMC.2010.53

Sablatash, M., 2007. Adaptive and cognitive UWB radio and a vision for flexible future spectrum management. Proceedings of the 10th Canadian Workshop on Information Theory, Jun. 6-8, IEEE Xplore Press, pp: 41-44.

DOI: 10.1109/CWIT.2007.375696
Salahdine, F., H. Ghazi, N. Kaabouch and W. Fihri, 2015. Matched filter detection with dynamic threshold for cognitive radio networks. Proceedings of the International Conference on Wireless Networks and Mobile Communications, Oct. 20-23, IEEE Xplore Press, pp: 1-6.

DOI: 10.1109/WINCOM.2015.7381345

Tarchi, D., A. Guidotti, V. Icolari, A. Vanelli-Coralli and S.K. Sharma et al., 2014. Technical challenges for cognitive radio application in satellite communications. Proceedings of the 9th International Conference on Cognitive Radio Oriented Wireless Networks and Communications, Jun. 2-4, IEEE Xplore Press, pp: 136-142. DOI: 10.4108/icst.crowncom.2014.255727

Xing, X., T. Jing, Y. Huo, H. Li and X. Cheng, 2013. Channel quality prediction based on bayesian inference in cognitive radio networks. Proceedings of the IEEE INFOCOM, Apr. 14-19, IEEE Xplore Press, pp: 1465-1473. DOI: 10.1109/INFCOM.2013.6566941

Yang, L., Z. Chen and F. Yin, 2012. Cyclo-energy detector for spectrum sensing in cognitive radio. Int. J. Electron. Commun., 66: 89-92.

DOI: $10.1016 /$ j.aeue.2011.05.004 